\title{
Disfunção da articulação sacro-ilíaca em jovens com dor lombar
}

\author{
Sacroiliac joint dysfunction in young adults with low back pain
}

\author{
Carolina Ramírez Ramírez ${ }^{[\mathrm{a}]}$, Diana Marina Camargo Lemus ${ }^{[\mathrm{b}]}$
}

[a] Mestre, professora da Escola de Fisioterapia da Universidade Industrial de Santander, Bucaramanga - Colômbia, e-mail: fisiocaro@yahoo.es

${ }^{[b]}$ Mestre, professora Escola de Fisioterapia da Universidade Industrial de Santander, Bucaramanga - Colômbia, e-mail: dcamargo@uis.edu.co

\section{Resumo}

Introdução: A dor lombar representa um problema de saúde pública. Estima-se que uma elevada porcentagem de casos de dor lombar (85-90\%) não tem uma causa conhecida. Acredita-se que a disfunção da articulação sacro-ilíaca possa explicar, em parte, essa alta porcentagem. Objetivo: Determinar a frequência da disfunção da articulação sacro-ilíaca em jovens com dor lombar e descrever possíveis associações com características sócio-demográficas, clínicas e antropométricas da população estudada. Metodologia: A amostra foi selecionada por meio de entrevista direta. Sessenta e sete sujeitos (92,5\% mulheres) com idade média de $21 \pm 2,1$ participaram do estudo. Foi realizado exame físico que incluiu a avaliação da dor à palpação, amplitude de movimento da flexão do quadril, funcionalidade avaliada por meio do Oswestry Disability Index, e realização de quatro provas de provocação de dor da articulação sacro-ilíaca (Gaenslen, thigh thrust, compressão ilíaca e compressão sacra). O diagnóstico para disfunção da articulação sacro-ilíaca foi considerado positivo se pelo menos três das provas foram positivas. Resultados: A frequência de disfunção da articulação sacro-ilíaca foi de 35,8\% IC 95\% $(24,0-47,6)$. Foi observada associação da disfunção da articulação com diminuição de flexibilidade dos isquiotibiais, pontuação do Oswestry Disability Index e dor à palpação. Não foram encontradas associações estatisticamente significativas com as variáveis sócio-demográficas. Conclusão: A alta frequência de disfunção da articulação sacro-ilíaca encontrada sugere a possibilidade de detectar e intervir precocemente uma possível causa de dor lombar, evitando alterações funcionais de maior severidade.

Palavras-chave: Articulação sacro-ilíaca. Dor lombar. Avaliação da incapacidade. 


\begin{abstract}
Intruduction: Low back pain (LBP) represents a public health problem. The etiology remains unknown in a high percentage of LBP (85-90\%) and it has been suggested that the sacroiliac joint dysfunction may be responsible for this high percentage. Purpose: To determine the frequency of sacroiliac joint dysfunction in young people with LBP and describe possible associations of the dysfunction with socio-demographic, clinical and anthropometric characteristics of the population. Methods: Subjects were selected by direct interview. Sixty seven subjects participated in the study $(92.5 \%$ women), with age mean of $21 \pm 2.1$ years. Physical examination consisted of pain evaluation, hip flexion range of motion, assessment of the functionality using the Oswestry Disability Index and reproduction of pain by four tests (Gaenslen, thigh thrust, iliac compression and sacrum compression). The diagnosis of sacroiliac joint dysfunction was made if at least three of the tests were positive. Results: The sacroiliac joint dysfunction frequency was $35.8 \%$ CI95\% (24.0-47.6). It was possible to observe association of sacroiliac joint dysfunction with the decreased hamstrings flexibility, Oswestry Disability Index score and pain during palpation. There were not significant associations between sacroiliac joint dysfunction and socio-demographic variables. Conclusion: The high sacroiliac joint dysfunction frequency suggests a possibility for early detection and treatment of a possible cause of LBP which may avoid functional alterations of greater severity.
\end{abstract}

Keywords: Sacroiliac joint. Low back pain. Incapacity assessment.

\title{
Introdução
}

A dor lombar (DL) representa um problema de saúde pública graças ao alto índice de afastamento, incapacidade e perda da produtividade do trabalhador (1-3). Apesar do uso de ferramentas diagnósticas como raios X, ressonância magnética e tomografia axial computarizada, e de uma adequada avaliação clínica, estimase que em 85\% das pessoas portadoras de DL não é possível determinar a etiologia da doença (4). Acredita-se que a Disfunção da Articulação Sacro-ilíaca (DASI) possa explicar, em parte, esta alta porcentagem $(5,6)$.

DASI é o termo utilizado para descrever a dor sobre ou ao redor da articulação sacro-ilíaca (ASI), provavelmente por mal alinhamento ou movimento anormal nesta articulação (5-7). Estudos recentes mostraram que entre 13-30\% das pessoas portadoras de DL apresentam DASI (7-10), portanto, este é um grupo populacional importante de ser investigado.

O diagnóstico clínico da DASI é complexo, em virtude das características anatômicas da articulação (como, por exemplo, a sua inervação) e pelo movimento restrito que possui $(8,9)$. Atualmente, considera-se que a prova de ouro para o diagnóstico de DASI seja o bloqueio anestésico intra-articular guiado por fluoroscopia $(5,9,11,12)$. No entanto, as provas de provocação de dor da ASI são reprodutíveis e válidas para diagnosticar essa disfunção (10,13-15). Apesar disso, faltam estudos que avaliem a frequência de DASI em populações de jovens utilizando provas de provocação de dor da ASI. O objetivo deste trabalho foi determinar a frequência da DASI mediante o uso de um grupo de provas de provocação de dor em jovens com DL, analisar uma possível associação com alguns fatores sócio-demográficos, antropométricos e clínicos, bem como analisar a concordância entre as provas utilizadas para o diagnóstico da DASI.

\section{Materiais e métodos}

\section{Sujeitos}

Foi realizado um estudo descritivo de tipo exploratório, no período de março a agosto de 2006. Na fase inicial, 175 estudantes de segundo ano de Fisioterapia (20,82 \pm 1,9 anos, 151 mulheres e 24 homens), selecionados por conveniência, preencheram o formulário de pré-seleção, fornecendo dados sócio-demográficos básicos e registrando a existência de dor lombar por meio de marcação da região da dor sobre um desenho 
corporal. Noventa e seis estudantes (58,4\%) relataram DL e foram convidados a participar do estudo. Foram considerados os seguintes critérios de inclusão: dor lombar (presença de dor entre L5 e a dobra glútea (16), dor há mais de quatro semanas de evolução (subaguda ou crônica), e intensidade igual ou superior a 3 na Escala Visual Analógica (EVA).

Foram excluídos os sujeitos em tratamento com anti-inflamatórios ou analgésicos, síndromes de dor crônica, fibromialgia, triggerpoint, doença de disco intervertebral diagnosticada (que tornaria conhecida a causa da DL), sinais de compressão nervosa (déficit sensitivo ou motor), presença do fenômeno de centralização $(17,18)$ (dor repetitiva na linha média da coluna, quando realizado o exame físico, que indicaria um resultado negativo nas provas usadas e possível lesão de disco), mulheres grávidas ou em período menstrual, pois a relaxina, hormônio produzido pelo corpo lúteo, gera lassitude ligamentosa e eventualmente dor na cintura pélvica, diferente da produzida pela DASI (19).

O estudo foi aprovado pelo Comitê de Ética de Pesquisa em Seres Humanos da universidade (Parecer n. 27650) e foi conduzido de acordo com a Declaração de Helsinki. Os sujeitos que cumpriram os critérios de inclusão e aceitaram participar do estudo assinaram o termo de consentimento livre e esclarecido.

\section{Avaliação}

Idade e sexo foram as variáveis sócio-demográficas avaliadas para verificação de possível associação com a DASI. Altura e peso também foram avaliados para o cálculo do Índice de Massa Corporal (IMC) com o objetivo de diferenciar os participantes com sobrepeso (IMC $>25$ ) de aqueles com peso normal (IMC $<25)$.

A experiência da dor foi caracterizada de acordo com o seu tempo de evolução (sub-aguda: 4-12 semanas; crônica: mais de 12 semanas) (4) e intensidade por meio da aplicação da EVA (ao repouso e à palpação bilateral da região glútea) com pontuação de 0 a $10 \mathrm{~cm}$, sendo que $0 \mathrm{~cm}$ significa ausência de dor e 10 $\mathrm{cm}$ a pior dor já sentida.

O exame físico foi composto de palpação a uma extensão de $10 \mathrm{~cm}$ abaixo e em sentido vertical, e $3 \mathrm{~cm}$ lateral à espinha ilíaca póstero-superior (EIPS) [(Sinal de Fortin (20)] para verificação de relato de dor. A flexibilidade dos isquiotibiais foi medida por meio do arco de movimento da flexão do quadril com joelho estendido (straight raise leg test), sendo que um movimento inferior a $70^{\circ}$ era indicativo de encurtamento da musculatura analisada (21). Para avaliar essa variável foi usado o goniômetro universal, o participante foi posicionado em supino com o membro não avaliado estendido sobre a maca. Enquanto isso, o membro avaliado foi elevado lentamente, mantendo o joelho em extensão até o avaliador perceber uma firme resistência e o participante confirmar que a flexão completa tinha sido atingida. O avaliador garantiu que o movimento do segmento avaliado fosse realizado unicamente no plano sagital (22).

A funcionalidade foi avaliada por meio da utilização do Oswestry Disability Index (ODI) que varia de 0 até 50, sendo que quanto maior a pontuação, maior a limitação funcional (23).

As provas de provocação de dor foram selecionadas com base em estudos prévios que obtiveram bons resultados de reprodutibilidade e validade $(5,10,13,14,24-26)$. As provas foram aplicadas em todos os sujeitos por uma fisioterapeuta com experiência na área ortopédica, seguindo as recomendações de Palmer et al. (27), Magge D (28), e Laslett et al. (13). As provas são descritas a seguir na mesma ordem de aplicação:

1) Prova de Gaenslen: sujeito em posição supina, no extremo inferior da maca, de forma que um dos membros inferiores pudesse ser hiperestendido. Flexionou-se o quadril e o joelho direito até o máximo possível, enquanto o esquerdo ficou estendido fora da maca. O examinador aplicou uma pressão nos joelhos no sentido dos movimentos antes descritos. A manobra foi repetida flexionando o membro esquerdo;

2) Compressão Ilíaca: com o sujeito em decúbito lateral, foram flexionados os quadris e os joelhos a $90^{\circ}$. O examinador, ajoelhado sobre a maca, aplicou uma força verticalmente em direção descendente sobre a região superior da crista ilíaca, mantendo os cotovelos estendidos. A prova foi realizada em decúbito lateral direto e esquerdo; 
3) Thigh thrust: o sujeito em supino, com abdução leve do quadril, flexão do quadril e do joelho em $90^{\circ}$, aplicou-se pressão em sentido vertical sobre o eixo longitudinal do fêmur. O procedimento foi realizado em cada membro inferior;

4) Compressão sacra: com o sujeito em decúbito prono, o avaliador pôs uma de suas mãos sobre a superfície posterior e medial do sacro, enquanto a outra mão foi colocada sobre a EIPS do lado avaliado. Aplicou-se uma força em sentido anterior e perpendicular, simultaneamente sobre as regiões mencionadas. A prova foi realizada sobre a EIPS direita e esquerda.

As provas foram consideradas positivas quando houve presença de dor na ASI ou na área glútea unilateral, e negativa quando houve dor na região lombar, centralização ou ausência de dor durante sua aplicação. O diagnóstico de DASI consistiu em registro positivo em três das quatro provas descritas $(13,14,18)$.

\section{Análise dos dados}

As variáveis foram descritas aplicando medidas de tendência central e dispersão ou tabelas de frequência, segundo a natureza e distribuição de cada variável. A avaliação de possíveis associações entre as variáveis do estudo com a presença de DASI realizou-se aplicando a prova de $\chi 2$ (vaiáveis medidas em escala nominal), e o teste t de Student (variáveis medidas em escala de razão) para grupos independentes. A concordância entre as provas foi avaliada mediante o Kappa de Cohen (29). A validação dos dados foi feita aplicando a rotina VALIDATE de Epi-Info 6,02. Após a validação dos dados, a análise estatística foi realizada utilizando-se o pacote estatístico Stata ${ }^{\circledR} 9.0(30,31)$. Foi considerada significância de $5 \%$.

\section{Resultados}

Foram entrevistados 175 estudantes na pré-seleção, e 96 deles (58,4\%) manifestaram DL. Dos 96 estudantes com DL, apenas 67 (21,1 $\pm 2,1$ anos, 62 mulheres e 5 homens) concluíram o estudo. Destes 67 participantes, 24 (35,8\% IC 95\% 24,0-47,6) foram diagnosticados com DASI, sendo que 22 foram mulheres $(91,6 \%)$ (Figura 1).

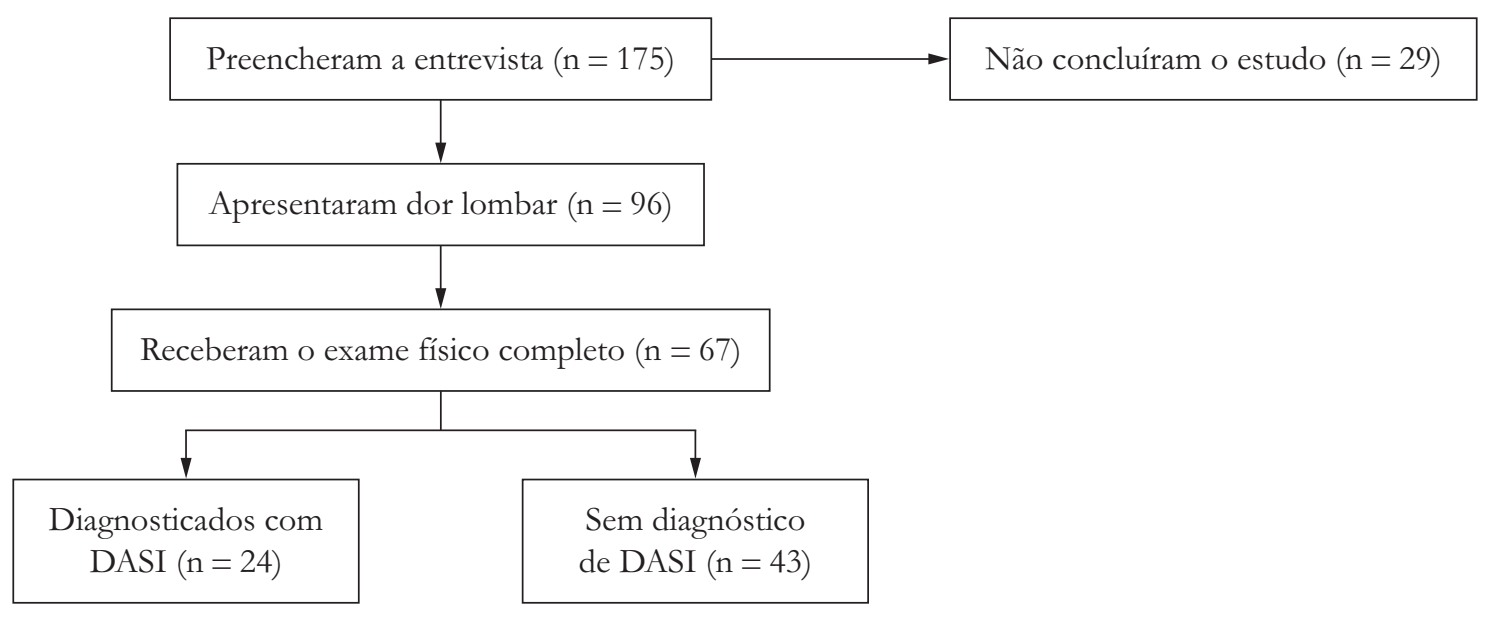

Figura 1 - Fluxograma apresentando as fases do estudo e o número de participantes. DASI= Disfunção da articulação sacro-ilíaca 
O IMC foi maior que $25 \mathrm{em} 12$ participantes, sendo que 50\% deles apresentaram DASI. A Tabela 1 apresenta os resultados do exame físico e as associações das variáveis do estudo segundo a presença da DASI.

A DL sub-aguda foi observada em três participantes $(4,5 \%)$ e a dor crônica foi registrada em 64 pessoas (95,5\%). Em 100\% dos indivíduos com DASI a DL foi do tipo crônica. A intensidade média de dor no EVA durante o repouso e a palpação foi de 5,4 e 6,3, respectivamente. Nos sujeitos com DASI, as pontuações na EVA foram superiores, quando comparadas com a ausência da disfunção (Tabela 1).

Os 67 sujeitos apresentaram encurtamento bilateral da musculatura isquiotibial $\left(57,1^{\circ} \pm 3,01\right)$. Foi encontrada uma pontuação de 9,6 \pm 6,1 quando aplicado o ODI, sendo que os portadores de DASI apresentaram maior pontuação, indicando maior limitação funcional quando comparados com sujeitos sem a disfunção (Tabela 1).

Os registros positivos das provas aplicadas mostraram que a prova de compressão sacra registrou a maior frequência, seguida pela prova de compressão lateral. Considerando a presença de DASI, a prova compressão sacra foi positiva em 100\% dos sujeitos com DASI, seguida pela thigh thrust e compressão ilíaca (Figura 2).

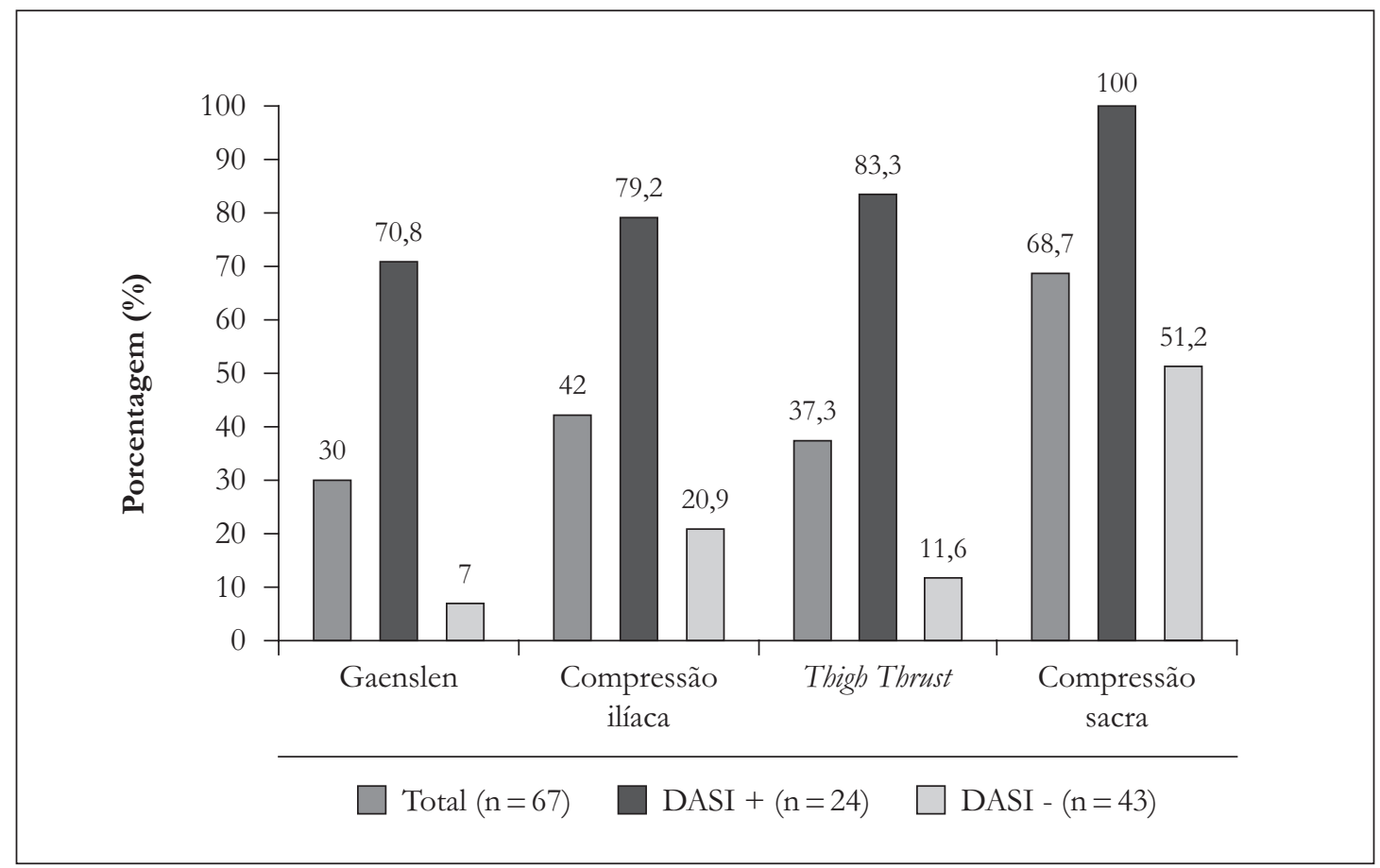

Figura 2 - Porcentagem de registros positivos nas provas de provocação de dor. Para cada prova é apresentada a porcentagem de registros positivos para a amostra total do estudo, para o grupo com (DASI+) e o grupo sem (DASI-) a disfunção

Ao avaliar a concordância entre as provas, foi encontrado Kappas entre 0,27 e 0,38. Os maiores valores foram observados para a prova compressão sacra com compressão ilíaca $(0,38)$, compressão sacra com thigh thrust $(0,37)$ e a prova de Gaenslen com a thigh thrust $(0,37)$, enquanto que as provas restantes tiveram Kappas inferiores a 0,30 .

Não foram encontradas associações estatisticamente significativas com a DASI quando analisadas as variáveis sócio-demográficas, tempo de evolução e intensidade da dor ao repouso. No entanto, houve associação significativa quando se considerou dor à palpação $(p=0,01)$, encurtamento bilateral da musculatura isquiotibial $(\mathrm{p}=0,03)$ e funcionalidade avaliada por meio da aplicação do ODI $(\mathrm{p}=0,02)$ (Tabela 1). 
Tabela 1 - Descrição da amostra e das possíveis associações das variáveis do estudo segundo a presença da DASI

\begin{tabular}{|c|c|c|c|c|}
\hline Variável & $\begin{array}{c}\text { Amostra } \\
\text { total }(n=67)\end{array}$ & $\begin{array}{c}\text { DASI }(+) \\
(n=24)\end{array}$ & $\begin{array}{c}\text { DASI }(-) \\
(\mathrm{n}=33)\end{array}$ & $p$ \\
\hline \multicolumn{5}{|l|}{$\operatorname{Sexo}(\mathbf{N})$} \\
\hline Feminino & $62(92,5 \%)$ & $22(91,6 \%)$ & 3 & 0,842 \\
\hline \multicolumn{5}{|l|}{ Idade (anos) } \\
\hline Média \pm DP & $21,1 \pm 2,1$ & $20,7 \pm 2,8$ & $21,2 \pm 2,1$ & 0,473 \\
\hline \multicolumn{5}{|l|}{$\operatorname{IMC}(\mathbf{N})$} \\
\hline$>25$ & 12 & 6 & 6 & 0,261 \\
\hline \multicolumn{5}{|l|}{ Evolução da DL (N) } \\
\hline Crônica & 64 & 24 & 40 & 0,191 \\
\hline Sub-aguda & 3 & 0 & 3 & \\
\hline \multicolumn{5}{|l|}{ EVA $(0-100)$} \\
\hline Repouso (Média \pm DP) & $5,4 \pm 1,9$ & $5,3 \pm 3,1$ & $4,8 \pm 1,6$ & 0,392 \\
\hline Palpação (Média \pm DP) & $6,3 \pm 1,5$ & $5,4 \pm 1,5$ & $4,2 \pm 1,8$ & $0,015^{*}$ \\
\hline \multicolumn{5}{|l|}{ ADM quadril (graus) } \\
\hline Direito (Média \pm DP) & $56,7 \pm 10,4$ & $53,1 \pm 8,0$ & $58,7 \pm 11,0$ & $0,032^{*}$ \\
\hline Esquerdo (Média $\pm D P)$ & $58,6 \pm 10,6$ & $54,2 \pm 90$ & $61,0 \pm 10,8$ & $0,010^{*}$ \\
\hline \multicolumn{5}{|l|}{ Pontuação ODI } \\
\hline Média \pm DP & $10,9 \pm 6,1$ & $13,5 \pm 5,4$ & $9,6 \pm 6,1$ & $0,021 *$ \\
\hline
\end{tabular}

$\mathrm{DP}=$ desvio padrão, $\mathrm{IMC}=$ Índice de massa Corporal, $\mathrm{ADM}=$ Amplitude de movimento, $\mathrm{EVA}=$ Escala Visual Analógica, ODI=Oswestry Index Disability.

* variável significativamente associada com a DASI.

\section{Discussão}

Para o nosso conhecimento, este é o primeiro trabalho a reportar a presença de DASI em um grupo de indivíduos jovens com DL. A frequência de DASI (35,8\%) encontrada no presente estudo foi similar à relatada em estudos prévios que utilizaram como diagnóstico a injeção intra-articular de anestésico guiada por fluoroscopia $(11,12,14,32)$. Isso sugere que é viável fazer o diagnóstico de DASI usando provas clínicas, as quais podem diminuir ou evitar a utilização de procedimentos invasivos, muitas vezes, desnecessários.

Considerando os resultados de concordância entre as provas deste trabalho, é possível sugerir que a utilização das provas compressão sacra, compressão ilíaca e thigh thrust seriam as mais adequadas para o diagnóstico fisioterapêutico de DASI. 
Embora outros autores não tenham analisado a correlação entre as provas, os resultados deste estudo poderiam se relacionar com maior prevalência, sensibilidade e valor preditivo negativo (VPN) para a thigh thrust (50\%, 0,88, 0,92, respectivamente) quando comparada com a injeção de anestésico (14). Laslett et al. (14) registraram também valores moderados de sensibilidade, especificidade, VPN e valor preditivo positivo (VPP) $(0,69 ; 0.69 ; 0,82$ e 0,52; respectivamente) para a prova de compressão ilíaca, bem como sensibilidade de 0,63, especificidade de 0,75 , VPN de 0,80 e VPP de 0,56 para a compressão sacra, quando comparada com a injeção de anestésico.

Neste trabalho, a prova de compressão sacra foi positiva em todos os indivíduos com diagnóstico de DASI, sendo também a prova com maior número de registros positivos nos sujeitos não portadores da disfunção (Figura 1). A alta frequência de registros positivos na prova de compressão sacra observada neste estudo pode ter sido influenciada pela não aleatorização na ordem de aplicação das provas, o que constitui uma possível limitação deste trabalho. Porém, Laslett et al. (14) aponta que a prova de compressão sacra, com a thigh thrust, possui uma influência forte sobre a capacidade diagnóstica de um grupo de provas de provocação de dor, quando se observa a área embaixo da Curva Operador Receptor (COR), a qual registrou um valor de 0,819 quando aplicadas essas duas provas em conjunto.

O critério para o diagnóstico de DASI foi baseado no registro positivo de três das quatro provas de provocação de dor, como previamente descrito $(13,14,18)$. Esses trabalhos compararam um grupo de provas de provocação de dor com a injeção intra-articular de anestésico e encontraram que três dessas provas positivas foram suficientes para o diagnóstico de DASI.

Nesse sentido, Laslett et al. (14) reportaram sensibilidade de 93,8\%, especificidade de 78,1\%, VPP 0,68, VPN 0,96 e área embaixo da COR de 0,842 quando comparou a injeção de anestésico, com a realização conjunta das provas de distração, thigh thrust, Gaenslen, compressão ilíaca e compressão sacra. Van-der-Wurff et al. (10) estudaram as provas de distração, compressão ilíaca, compressão sacra, Gaenslen, Patrick-FABER e thigh thrust, e relataram que quando três ou mais destas provas foram positivas, verificou-se probabilidade de $65-93 \%$ de que a origem da dor fosse a ASI. Os mesmos autores também demonstraram sensibilidade de 0,85; especificidade de 0,79; VPP 0,77; VPN 0,87 e área embaixo da COR de 0,77, quando o diagnóstico é realizado considerando três dessas provas positivas.

Os trabalhos anteriores apoiam o uso das provas aplicadas neste estudo na prática fisioterapêutica cotidiana. Quando essas provas são realizadas por profissionais treinados, há possibilidade de realizar um diagnóstico correto (15), e isso pode diminuir a realização de procedimentos invasivos.

A associação positiva da DASI com a dor à palpação observada neste trabalho está de acordo com os achados de Fortin et al. (20), os quais reportaram reprodutibilidade excelente interavaliadores (Kappa $=$ $0,96)$ para a presença deste sinal em indivíduos com DASI, bem como VPP de 0,87 para o diagnóstico de doença na ASI, quando comparado com a injeção intra-articular de anestésico.

O IMC não mostrou associação com a presença de DASI, possivelmente por causa do pequeno número de indivíduos com sobrepeso $(\mathrm{n}=12)$. Esses resultados coincidem com o trabalho de Irwin et al. (33), que também não encontraram associação da DASI com a idade e o IMC. No entanto, a idade média dos sujeitos avaliados no estudo citado foi de 53,31 anos. Entretanto, é interessante observar que no presente estudo 50\% dos sujeitos com sobrepeso apresentaram DASI. Isso poderia sugerir que o aumento de peso sobrecarrega a coluna lombar e a região pélvica, predispondo à maior degeneração articular (34), aumentando desta forma a probabilidade de desenvolver DASI.

O encurtamento dos isquiotibiais esteve presente em 100\% dos participantes com DASI. Isso é interessante, já que a população deste estudo foi composta por indivíduos jovens e ativos. Para Calliet (35), o encurtamento dos isquiotibiais é um dos fatores que podem contribuir com a DL, devido à inserção e a sua influência no ritmo lumbo-pélvico. De outra parte, Kendall (36) afirma que isquiotibiais encurtados rodam a pelve em sentido posterior, reduzem a curvatura lombar, alteram o ritmo lumbo-sacro e geram um movimento compensatório na região lombar, e isso poderia sobrecarregar essa área e desencadear DL. Sendo assim, pode-se sugerir que a mudança no movimento do ilíaco afetaria a mecânica da ASI, alterando sua habilidade para absorver forças de reação do solo e de diminuir o cisalhamento sobre os discos intervertebrais durante a marcha (37). Deste modo, a DASI elevaria a carga sobre a região lombar, gerando uma potencial DL. 
Os resultados do ODI mostraram limitação funcional de leve a moderada nos indivíduos com DASI. Segundo Fairbank et al. (23), essa pontuação equivale a 21\% de incapacidade. Estes resultados demonstram o impacto que a DASI gera na funcionalidade de pessoas jovens. A associação significativa da limitação funcional com a DASI registrada neste estudo poderia estar relacionada com a presença simultânea de dor crônica, a qual tem mostrado impacto negativo sobre a funcionalidade das pessoas com esta condição (38).

Para o nosso conhecimento ainda não foi demonstrado na literatura a associação da DASI com a limitação funcional. Alguns autores descreveram somente a pontuação obtida com instrumentos de avaliação funcional, como o Roland-Morris 14 e o ODI (10), sem analisar a relação entre as variáveis. Portanto, este estudo é o primeiro a descrever essa associação. A associação positiva registrada neste trabalho fornece subsídios sobre a importância da DASI na função nas etapas precoces da vida adulta e sobre a importância da Fisioterapia na identificação e intervenção precoce desta condição, diminuindo, assim, o impacto negativo na qualidade de vida dos sujeitos com DASI em períodos tardios da vida adulta.

Uma das limitações deste trabalho é a homogeneidade da amostra estudada. Esse fato pode ter restringido a associação da DASI com algumas características sócio-demográficas analisadas. Por isso, a caracterização da DASI no âmbito populacional precisa de estudos com uma amostra maior, que permita determinar a sua prevalência, bem como a presença de fatores associados, fornecendo subsídios para o planejamento e execução de programas preventivos e terapêuticos.

\section{Conclusão}

A frequência da DASI registrada neste estudo indica a necessidade de diagnosticar e intervir de forma precoce numa potencial causa de DL, visando a melhorar a qualidade de vida das pessoas portadoras dessa disfunção. Dentre os fatores associados destacam-se o encurtamento da musculatura isquiotibial e a limitação funcional dos sujeitos portadores de DASI. De outro lado, o presente estudo sugere a utilidade das provas de provocação de dor da ASI como método de diagnostico clínico na prática fisioterapêutica cotidiana.

\section{Referências}

1. Strine TW, Hootman JM. US national prevalence and correlates of low back and neck pain among adults. Arthritis Rheum. 2007;57(4):656-65.

2. Hemingway H, Shipley M, Stansfeld S, Shannon H, Frank J, Brunner E, et al. Are risk factors for atherothrombotic disease associated with back pain sickness absence? The Whitehall II Study. J Epidemiol Community Health. 1999;53(4):197-203.

3. Meerding WJ, IJzelenberg W, Koopmanschap MA, Severens JL, Burdorf A. Health problems lead to considerable productivity loss at work among workers with high physical load jobs. J Clin Epidemiol. 2005;58(5):517-23.

4. Riddle DL. Classification and low back pain: a review of the literature and critical analysis on selected systems. Phys Ther. 1998;78(7):708-37.

5. Freburger JK, Riddle DL. Using published evidence to guide the examination of the sacroiliac joint region. Phys Ther. 2001;81(5):1135-43.

6. Freburger JK, Riddle DL. Evaluation of the presence of sacroiliac joint region dysfunction using a combination of tests: a multicenter intertester reliability study. Phys Ther. 2002;82(8):772-81.

7. O'Sullivan PB, Beales SJ, Beetham JA. Altered motor control strategies in subjects with sacroiliac joint pain during the active straight leg raise test. Spine. 2002;27(1):E1-8. 
8. Zelle BA, Gruen GS, Brown S, George S. Sacroiliac joint dysfunction: evaluation and management. Clin J Pain. 2005;21(5):446-55.

9. Cohen PS. Sacroiliac joint pain: a comprehensive review of anatomy, diagnosis and treatment. Anesth Analg. 2005;101(5):1440-53.

10. Van-der-Wurff P, Buijs EJ, Groen GJ. A multitest regimen of pain provocation test and aid to reduce unnecessary minimally invasive sacroiliac joint procedures. Arch Phys Med Rehabil. 2006;87(1):10-4.

11. Schwazer AC, Aprill C, Bogduk N. The sacroiliac joint in cronic low back pain. Spine. 1995;20(1):31-7.

12. Maigne JY, Aivaliklis A, Pfefer F. Result of sacroiliac joint double blockade and value of sacroiliac pain provocation tests in 54 patients with low back pain. Spine. 1996;21(16):1889-92.

13. Laslett M, Young SB, April C. Diagnosing painful sacroiliac joints: a validity study of a Mackenzie evaluation and sacroiliac provocation tests. J Aust Physiother. 2003;49:89-97.

14. Laslett M, Aprill C, McDonald B, Young S. Diagnosis of Sacroiliac Joint Pain: validity of individual provocation test and composites of test. Man Ther. 2005;10(3):207-18.

15. Robinson HS, Brox IJ, Robinson R, Bjelland E, Solem S, Telje T. The realibility of selected motion-and pain provocation tests for the sacroiliac joint. Man Ther. 2007;12(1):72-9.

16. Papageorgiou AC, Croft PR, Ferry S, Jayson MI, Silman AJ. Stimating the prevalence of low back pain in the general population: evidence from the South Manchester Back Pain Survey. Spine. 1995;20(17): 1889-94.

17. McKenzie RA, May S. Mechanical diagnosis and therapy: the lumbar spine. 2nd ed. New Zeland: Spinal Publication; 2002.

18. Laslett M, Oberg B, Aprill C, McDonald B. Centralization as a predictor of provocation discography results in chronic low back pain, and the influence of disability and distress on diagnostic power. Spine. 2005;5(4):370-80.

19. Walker J. The sacroiliac joint: a critical review. Phys Ther. 1992;72(12):903-16.

20. Fortin JD, Dweyr AP, West S, Pier J. Sacroiliac joint: pain referral maps upon applying a new injection/arthroraphy techinique. Part I: clinical evaluation. Spine. 1994;19(13):1475-82.

21. Li Y, McClure P, Pratt N. The effect of hamstrings muscle stretching on standing posture and on lumbar and hip motion during forward bending. Phys Ther. 1996;76:836-45.

22. Stuelcken MC, Ginn KA, Sinclair PJ. Musculoskeletal profile of the lumbar spine and hip regions in cricket fast bowlers. Phys Ther Sport. 2008;9(2):82-8.

23. Fairbank, JC, Couper J, Davies JB, O'Brien JP. The Oswestry low back pain disability questionnaire. Physiotherapy. 1980;66(8):271-3.

24. Potter NA, Rothstein JM. Interrester reliability for selected clinical tests of the sacroiliac joint. Phys Ther. 1985;65(11):1671-5.

25. Van-der-Wurff P, Hagmeijer, Mayne W. Clinical tests of the sacroiliac joint: a systematic methodological review. Part I: Reliability. Man Ther. 2000;5(1):30-6.

26. Van-der-Wurff P, Hagmeijer, Mayne W. Clinical tests of the sacroiliac joint. A systematic methodological review. Part 2: Validity. Man Ther. 2000;5(2):89-96.

27. Palmer M, Epler M. Musculoskeletal assesment techniques. 2nd ed. New York: Lippincott; 1998.

28. Magge D. Orthopaedic physical assesment. 4th ed. Philadelphia: Sanders; 2002. 
29. Streiner DL, Norman GR. Health measurement scales: a practical guide to their development and use. New York: Oxford University Press; 2003.

30. Dean AG, Dean JA, Coulumbier D, Brendel KA, Smith DC, Burton AH, et al. Epi Info, Version 6.01: a word processing, database, and statistics program for epidemiology on microcomputers. Georgia: Center for Disease Control and Prevention; 1995.

31. Siegel S. Nonparametric statistics for the behavioral sciences. 2nd ed. New York: McGraw Hill; 1989.

32. Hodges JC, Besset B. The incidence of sacroiliac joint disease in patients with low back pain. Can Assoc Radiol J. 1999;50(5):321-3.

33. Irwin RW, Watson T, Minick RP, Ambrosius WT. Age, body mass index and gender differences in sacroiliac joint pathology. Am J Phys Med Rehabil. 2007;86(1):37-44.

34. Faflia CP, Prassopoulos PK, Daskalogiannaki ME, Gourtsoyiannis NC. Variation in the appearance of the normal sacroiliac joint on pelvic. Clin Radiol. 1998;53(10):742-6.

35. Cailliet R. Low Back Pain Syndrome. 5th ed. Phyladelphia: F.A. Davis Company; 1995.

36. Kendal FP, McCreary EK. Muscles: testing and functions. 5th ed. Philadelphia: Lippincot; 2005.

37. DonTigny RL. Anterior dysfunction of the sacroiliac joint as a major factor in the etiology of Idiophatic Low Back Pain Syndrome. Phys Ther. 1990;70(4):250-65.

38. Breivik H, Collett B, Ventafridda V, Cohen R, Gallacher D. Survey of chronic pain in Europe: prevalence, impact on daily life, and treatment. Eur J Pain. 2006;10(4):287-333.

Recebido: 19/03/2009

Received: 03/19/2009

Aprovado: 28/03/2010

Approved: 03/28/2010

Revisado: 24/06/2010

Reviewed: 06/24/2010 